\title{
Análise métrica das marcas de mordidas impressas em alimentos acondicionados em diferentes temperaturas: perspectivas periciais de um estudo piloto
}

Metric analysis of bite marks printed on food packaged at different temperatures: the expert perspectives of a pilot study

Análisis métrico de las marcas de mordeduras impresas en alimentos acondicionados a diferentes temperaturas: perspectivas periciales de un estudio piloto

\author{
Cléa Adas Saliba GARBIN ${ }^{1}$ \\ Jeidson Antônio Morais MARQUES ${ }^{2}$ \\ Naiana de Melo BELILA ${ }^{3}$ \\ Artênio José Isper GARBIN ${ }^{\mathbf{1}}$
}

${ }^{I}$ Departamento de Odontologia Infantil e Social, Universidade Estadual Paulista (UNESP), Faculdade de Odontologia de Araçatuba 16015-050 Araçatuba-SP, Brasil ${ }^{2}$ Departamento de Saúde da Universidade Estadual de Feira de Santana (UEFS) 44036-900 - Feira de Santana-BA, Brasil

${ }^{3}$ Doutoranda, Programa de Pós-Graduação em Odontologia Preventiva e Social, Universidade Estadual Paulista (UNESP), Faculdade de Odontologia de Araçatuba 16015-050 Araçatuba-SP, Brasil

\section{Resumo}

Introdução: Existe uma área significativa de estudos e análises no campo da Odontologia Forense, que é o reconhecimento e a interpretação de sinais e lesões produzidas por mordidas humanas, que podem ser observadas tanto na pele como em alimentos. Objetivo: Avaliar a técnica da análise métrica das impressões dentárias em alimentos utilizados como prova pericial, bem como a melhor forma de conservação desses alimentos mordidos de acordo com a variação de temperatura e a influência do tempo para a identificação do autor. Metodologia: Estudo do tipo duplo-cego. Realizou-se um sorteio, obtendo uma amostra de quatro indivíduos. Para cada um, foi solicitado que mordesse uma barra de chocolate e uma goma de mascar, usando apenas os dentes anteriores. Os alimentos coletados foram divididos em 2 grupos e acondicionados em recipientes plásticos hermeticamente fechados, sob temperaturas de $4^{\circ} \mathrm{C}$ e o outro sob $-10^{\circ} \mathrm{C}$. Com o uso de um paquímetro digital, realizaram-se mensurações das marcas impressas nos alimentos a cada três meses, durante um ano. Resultados: As marcas equivalentes a cada dente por meio da Análise Métrica verificada a cada três meses, não apresentaram variações significativas ( $p>0,05$ ). Apesar das diferentes temperaturas estudadas e do tempo transcorrido, as proporções foram mantidas, não comprometendo a investigação e possibilitando a identificação dos autores com $100 \%$ de êxito. Conclusão: A técnica da análise métrica das impressões dentárias em alimentos e o acondicionamento a baixas temperaturas, mesmo após o tempo de um ano transcorrido, preservou as proporções entre as marcas desejadas por cada dente.

Descritores: Odontologia Legal; Violência; Prova Pericial.

\section{Abstract}

Introduction: There is a significant area of study and analyze in the field of Forensic Dentistry, which is the recognition and interpretation of signs and lesions produced by human bites, which can be observed both in the skin and in food. Objective: To evaluate the technique of metric analysis of dental impressions in foods used as criminal evidence, as well as the best way of preserving these foods according to temperature variation and the influence of time for the identification of the author of the crime. Methodology: A double-blind study. A lottery was carried out, obtaining a sample of four individuals. Each of them was asked to bite a bar of chocolate and a chewing gum, using only the front teeth. The collected foods were divided into 2 groups and packed in hermetically sealed plastic containers under temperatures of $4{ }^{\circ} \mathrm{C}$ and the other under $-10^{\circ} \mathrm{C}$. With the use of a digital caliper, markings were measured on foods every three months in the period of a year. Results: The marks equivalent to each tooth unit by means of the Metric Analysis was verified every three months and did not present significant variations $(p>0.05)$. Despite the different temperatures studied and the time elapsed, the proportions were maintained, not compromising the investigation and allowing the identification of the authors of the crime with $100 \%$ success. Conclusion: The technique of the metric analysis of dental impressions in food and the preservation at low temperatures, even after one year, preserved the proportions between the marks of each tooth.

Descriptors: Forensic Dentistry; Violence; Expert Testimony.

\section{Resumen}

Introducción: Existe un área significativa de estudios y análisis en el campo de la Odontología Forense, que es el reconocimiento y la interpretación de señales y lesiones producidas por mordeduras humanas, que pueden ser observadas tanto en la piel como en los alimentos. Objetivo: Evaluar la técnica del análisis métrico de las impresiones dentales en alimentos utilizados como prueba pericial, así como la mejor forma de conservación de esos alimentos mordidos de acuerdo con la variación de temperatura y la influencia del tiempo para la identificación del autor. Metodología: Estudio del tipo doble ciego. Se realizó un sorteo, obteniendo una muestra de cuatro individuos. Para cada uno, se le pidió que mordiera una barra de chocolate y una goma de mascar, usando sólo los dientes anteriores. Los alimentos recolectados fueron divididos en 2 grupos y acondicionados en recipientes plásticos herméticamente cerrados, a temperaturas de $4^{\circ} \mathrm{C}$ y el otro bajo $-10^{\circ} \mathrm{C}$. Con el uso de un paquímetro digital, se realizaron mediciones de las marcas impresas en los alimentos cada tres meses, durante un año. Resultados: Las marcas equivalentes a cada diente por medio del Análisis Métrico verificado cada tres meses, no presentaron variaciones significativas $(\mathrm{p}>0,05)$. A pesar de las diferentes temperaturas estudiadas y del tiempo transcurrido, las proporciones se mantuvieron, no comprometiendo la investigación y posibilitando la identificación de los autores con el 100\% de éxito. Conclusión: La técnica del análisis métrico de las impresiones dentales en alimentos y el acondicionamiento a bajas temperaturas, incluso después del tiempo de un año transcurrido, preservó las proporciones entre las marcas deseadas por cada diente.

Descriptores: Odontología Forense; Violencia; Testimonio de Experto.

\section{INTRODUÇÃO}

A perícia Odontológica consiste em todo procedimento de investigação científica, solicitado por autoridade policial ou judiciária, sendo praticada pelo cirurgião dentista. Este, por meio de seu conhecimento especializado, é capaz de esclarecer à justiça diversas questões no âmbito criminal, civil, trabalhista e administrativo. $\mathrm{O}$ perito deve ter $\mathrm{o}$ entendimento de que a identificação Odonto e Médico-Legal é baseada em uma coleção de dados que servem de apoio técnico na elucidação de casos $^{1,2}$.

O cenário atual mostra um crescimento da violência, fazendo com que os crimes se tornem cada vez mais sofisticados, exigindo assim o 
desenvolvimento de técnicas periciais mais aperfeiçoadas e aprimoradas ${ }^{1,2}$.

Dentre as marcas de agressões, existe uma área significativa de estudos e análises no campo da Odontologia Forense, que é o reconhecimento e a interpretação de sinais e lesões produzidas por mordidas humanas e que podem ser observadas tanto na pele como em alimentos. Dentre eles, os registros são encontrados em queijos, chocolates, maças, laranjas, pepinos, biscoitos, lanches, goma de mascar, tortas, entre outros ${ }^{3}$.

A impressão da mordida é definida pela American Board of Forensic Odontology $(\mathrm{ABFO})^{4}$, como uma marca de agressão, circular ou oval consistindo em dois arcos em forma de U, opostos e simétricos, separados em suas bases por espaços; em sua periferia podem existir abrasões, contusões e/ou lacerações que refletem o tamanho, forma $\mathrm{e}$ localização das superfícies de contato da dentição humana.

A identificação das marcas de mordida é baseada na característica individualista da dentição, onde a ligação entre o agressor e a marca é fundamentada na comparação dente a dente e arco a arco, utilizando os parâmetros de tamanho, forma e alinhamento ${ }^{5}$.

O processo de identificação da marca de mordida é complexo e requer um bom conhecimento e experiência por parte do perito odontolegista, tornando a participação de um cirurgião dentista essencial no processo de identificação dessas marcas ${ }^{6}$.

Nesse contexto, o presente estudo teve por objetivo avaliar a técnica da análise métrica das impressões dentárias em alimentos utilizados como prova pericial, bem como a melhor forma de conservação desses alimentos mordidos de acordo com a variação de temperatura e a influência do tempo para a identificação do autor.

\section{MATERIAL E MÉTODO}

A pesquisa consiste em um estudo do tipo duplo-cego, onde foi teve o seu universo populacional composto por 50 indivíduos, que eram graduandos da Faculdade de Odontologia da Universidade de São Paulo. Como critérios de inclusão, os participantes deveriam ter uma boa saúde bucal, com menor número de dentes cariados possível, fraturas e ausência de dentes.

Esses alunos foram moldados (Alginato Hydrogum, Zhermack) e assim, obtidos 50 modelos em gesso (Gesso Pedra Tipo III G3, Durastone) para a realização da pesquisa.

A coleta da amostra foi dividida em duas etapas. Na primeira, foi realizado um sorteio, onde foram selecionados quatro indivíduos da população alvo, compondo assim a amostra do estudo. Para cada um, foi solicitado que mordesse uma barra de chocolate (Lacta, Mondelez Brasil Ltda.), usando para isso apenas os dentes anteriores, de canino a canino. A etapa seguinte processou-se da mesma forma, porém utilizaram-se quatro gomas de mascar (Trident, Mondelēz International, Inc.) para os registros. Apesar de geralmente as gomas de mascar serrem mordidas com os dentes posteriores, neste estudo foram utilizadas para verificar a conservação dos alimentos e não a impressão propriamente dita. As impressões dentárias foram obtidas de forma cega para o investigador.

Após os alimentos serem coletados, foram divididos em 2 grupos (onde cada um possuía 2 barras de chocolates e 2 gomas de mascar) e acondicionados em recipientes plásticos hermeticamente fechados (Saco Zip Lock com Fecho Hermético), onde um grupo ficou sob temperatura de $4^{\circ} \mathrm{C}$ e o outro sob $-10^{\circ} \mathrm{C}$. Com o uso de paquímetro digital (Mitutoyo) foram realizadas mensurações das marcas impressas nos alimentos e este procedimento foi repetido a cada três meses, durante um ano para verificar supostas alterações nos alimentos.

Depois desse período, a partir dos modelos em gesso e dos alimentos mordidos pelos quatro participantes, foi utilizada uma metodologia de identificação de marcas de mordidas para relacionar cada um dos indivíduos com seus respectivos alimentos ${ }^{4}$. Foi realizada a moldagem dos alimentos com silicona de condensação e desta forma, obtevese o modelo em gesso das impressões dentárias.

A técnica utilizada foi a de Análise Métrica baseada nas Normas da $\mathrm{ABFO}^{5}$, ou seja, todos os detalhes das marcas foram medidos e registrados. Foram calculados: o comprimento, largura e a profundidade das marcas de cada dente específico, a forma do local da impressão e outras dimensões; como a distância intercanina, o espaço entre as marcas dos dentes, indicadores de maloclusão e ausência de dentes.

Depois de obtidas as medidas com um paquímetro digital, foram efetuadas comparações métricas equivalentes a cada dente e as que, em conjunto coincidiram, passaram por uma comparação direta com a cópia da impressão da mordida, em gesso. O paquímetro foi utilizado devido a sua disponibilidade em laboratórios periciais com pouca infraestrutura e baixo custo. Foi utilizado para a avaliação das distâncias intercaninas, o teste estatístico "T" Student.

Esta pesquisa foi aprovada pelo Comitê de Ética em Pesquisa em Seres Humanos da Faculdade de Odontologia da Universidade de São Paulo (Protocolo N.58/2003) e os indivíduos que concordaram em participar assinaram o Termo de Consentimento Livre e Esclarecido.

RESULTADOS

Os resultados mostraram que as marcas 
equivalentes a cada dente por meio da Análise Métrica verificada a cada três meses, não apresentaram variações significativas $(p>0,05)$.

Apesar das diferentes temperaturas estudadas $\left(4^{\circ} \mathrm{C} \mathrm{e}-10^{\circ} \mathrm{C}\right)$ e do tempo transcorrido (um ano), nos dois grupos foram preservadas as proporções entre as marcas desejadas por cada dente. Desta forma, não houve diferença estatística significativa entre as gomas de mascar e as barras de chocolate, porém, os chocolates apresentaram-se mais friáveis, cabendo a atenção do perito e cuidado durante o manuseio desses alimentos durante o armazenamento $\mathrm{e}$ resfriamento.

Apesar da distorção observada, as proporções foram mantidas, não comprometendo a investigação dos alimentos e possibilitando a identificação dos autores, por meio da Análise Métrica, com 100\% de êxito.

\section{DISCUSSÃO}

A importância dos estudos odontolegais, dentro do instituto Médico-Legal, está se tornando cada vez mais evidente, efetiva e apresentando um inquestionável valor legal. $\mathrm{O}$ perito odontolegal executa funções tais como: auxilia na identificação de corpos submetidos a grandes catástrofes; na identificação de criminosos pelo reconhecimento da mordida; se dá a estimativa da idade, estatura, raça, dano estético e funcional do indivíduo; e pode atribuir a culpa da agressão a um determinado suspeito ou a sua exclusão do mesmo, sendo capaz de solucionar diversos tipos de crimes e agressões ${ }^{6,8-10}$.

No campo criminal, um dos desafios mais complexos e intrigantes é a conservação dos registros das análises das marcas de mordidas, que têm sido investigadas tanto na pele como em vários alimentos ${ }^{11}$. Devido ao grande número de casos envolvendo esse tipo de lesão, houve um gradual aumento no número de peritos odontolegais dentro dos institutos criminais ${ }^{12}$.

O princípio da base científica da análise das impressões dentárias é estabelecido pela individualidade da arcada dentária. Autores afirmam que não existem dois indivíduos com as mesmas características na dentição, dada a enorme variedade de particularidades individuais proporcionadas pelas peças dentárias ${ }^{13}$. Em 1984, Rawson e colaboradores analisaram estatisticamente a possibilidade de dois arcos serem iguais, onde o cálculo mostrou que a probabilidade de dois conjuntos de dois coincidirem é de 1 em 384 arcos; sendo ressaltado que nessa pesquisa não foram avaliadas as fraturas, perdas dentárias, restaurações, entre outros fatores que asseguram ainda mais a unicidade da mordida humana ${ }^{14}$. No entanto, em 2011, Bush reproduziu o estudo anterior, encontrando resultados contrários, considerando essa regra inapropriada ${ }^{15}$.

Fato que levou a escolha da amostra neste estudo, baseado no fato de que os alunos de Odontologia possuíam uma boa saúde bucal, sendo consideravelmente menor o número de dentes cariados, fraturas e ausência de dentes. Estas características tornam os arcos dentários mais individuais e assim, possibilita identificar o autor da mordida, havendo uma maior credibilidade durante a identificação, pois, geralmente, os casos policiais possuem poucos suspeitos.

Nesta pesquisa, os tipos de materiais para a obtenção das mordidas foram escolhidos devido à frequência em que são encontrados em cenas criminalísticas.

As gomas de mascar, apesar de ser pouco utilizada na identificação de criminosos, de acordo com o estudo de Nambiar ${ }^{16}$, são os alimentos que proporcionaram a identificação de forma mais rápida e segura, sendo provavelmente o único alimento a registrar com detalhes as superfícies oclusais dos dentes posteriores. Estudos mostram que alimentos pegajosos como as gomas de mascar podem distorcer os registros dentários não permitindo o registro físico, desta forma, a identificação pode ser realizada por meio de evidências biológicas, utilizando a saliva para determinar o grupo sanguíneo do indivíduo e extração do DNA, permitindo-se identificar o perfil molecular do mesmo ${ }^{7,16}$.

No entanto, neste estudo a goma de mascar mostrou-se um alimento confiável por não ter sofrido alterações ou distorções quando armazenada de forma correta e possibilitando desta forma o reconhecimento das arcadas dentárias.

Outro método utilizado na identificação das marcas de mordidas é a Análise Métrica, onde cada detalhe ou traço do dente do suspeito que é capturado na lesão deve ser medido e registrado. O comprimento, a largura e a profundidade das marcas de cada dente específico; a dimensão e a forma do local da injúria e outras dimensões como a distância intercanina, espaço entre as marcas dos dentes, indicações de mau posicionamento ou ausência de dentes devem ser registrados e calculados. As medidas (em milímetros) das particularidades podem ser notificadas de acordo com o escore preconizado pela $\mathrm{ABFO}^{5,7}$.

A Análise Métrica nesta pesquisa mostrou-se um método eficiente para verificar a inexistência de deformações nos alimentos do estudo. A técnica foi realizada a cada três meses, proporcionando a comparação entre as medições realizadas durante um ano.

Por sua vez, é caracterizada como a técnica de maior simplicidade, podendo ser utilizada em laboratórios que não dispõe de equipamentos sofisticados e de custo elevado. Esta realidade é bastante presente em países subdesenvolvidos ou em desenvolvimento como, por exemplo, o Brasil. O avanço tecnológico modifica significativamente os 
métodos de análise das impressões dentárias, como o uso de tomografias computadorizadas, tecnologias de softwares, fotografias, mas não dispensam a análise métrica da mordida ${ }^{17}$. Nos casos em que existe a presença de alimentos, conforme este estudo, a análise direta dos modelos de gesso, é um método mais seguro para a identificação de criminosos.

A escolha das temperaturas utilizadas neste estudo (4 e $\left.-10^{\circ} \mathrm{C}\right)$, baseou-se na compatibilidade com as encontradas em geladeiras de uso doméstico com o intuito de minimizar a deformação dos alimentos pelo calor e que fossem mantidas as características do alimento durante o estudo ${ }^{18,19}$.

Nas temperaturas estudadas, as gomas de mascar mostraram confiáveis, pois nas duas variações não sofreram nenhuma distorção. Já as barras de chocolate tornaram-se mais friáveis, porém, este fato não impossibilitou o reconhecimento das arcadas, validando assim que as temperaturas estudadas são eficientes para manter a integridade dos alimentos.

Os arcos dentários vêm se tornando um importante meio na identificação de criminosos, principalmente pela diversidade dos elementos que são coletados na amostra e que carregam um valor imensurável na comprovação de um delito. As características individuais de cada elemento dentário, combinado com as estruturas periféricas, dão um caráter único a cada mordida ${ }^{20}$.

Desta forma, os alimentos estudados nesta pesquisa mostraram-se subsídios imprescindíveis no auxílio de uma investigação, onde $o$ perito odontolegal deve estar atento para a coleta desses materiais quando disponíveis em cenas de crimes.

\section{CONCLUSÃO}

Conclui-se que a técnica da análise métrica das impressões dentárias em alimentos e o acondicionamento a baixas temperaturas, tanto das gomas de mascar como das barras de chocolates, mesmo após o tempo de um ano transcorrido, preservou as proporções entre as marcas desejadas por cada dente. Desta forma, o método mostrou-se eficiente, possibilitando a identificação dos autores correspondentes.

\section{REFERÊNCIAS}

1. Galvão LCC. Estudos médicos legais. Porto Alegre: Sagra; 1996.

2. Amorim HPL, Melo BMS, Musse JO, Silva MLCA, Costa COM, Marques JAM. Levantamento de marcas de mordidas humanas em vítimas de violência periciadas no Instituto Médico Legal de Feira de Santana-BA, entre 2007 e 2014. Arq Odontol. 2016;52(3):165-74.

3. Coutinho CGV, Ferreira CA, Queiroz LR, Gomes LO, Silva UA. O papel do odontolegista nas perícias criminais. RFO UPF. 2013;18(2):217-23.

4. Nascimento MM, Sarmento VA, Beal VE, Galvão LCC, Marques JAM. Identificação de indivíduos por meio das marcas de mordida em alimentos utilizando a engenharia reversa e a prototipagem rápida: caso simulado. Arq Odontol. 2012;48(3):134-41.

5. American Board of Forensic Odontology, Inc. Diplomates Reference Manual Section I: Preface, Acknowledgments, Background, Functions \& Purposes. March 2017 Edition.

6. Pretty IA, Hall RC. Forensic dentistry and human bite marks: issues for doctors. Hosp Med. 2002; 63(8):476-82.

7. Goetten FS. Avaliação de lesões corporais em vítimas de mordeduras: uma revisão da literatura. Rev Bras Crimin. 2014;3(2):41-5.

8. Melani RFH. Marcas de mordidas. In: Silva M. Compêndio de Odontologia Legal. São Paulo: Medis; 1997.

9. França G. Medicina Legal. 7. ed. Rio de Janeiro: Guanabara Koogan; 2004.

10.Garbin CAS, Lima TJV, Garbin AJI, Rovida TAS, Saliba O. Conhecimento e percepção dos educadores do ensino infantil sobre violência. Ciência Plural. 2015;1(2):37-47.

11. Wright FD, Dailey JC. Human bite marks in forensic dentistry. Dent Clin North Am. 2001;45(2):365-97.

12.Santos KRR, Pereira PZ. Verificação do potencial de atuação do cirurgião-dentista nas necrópsias realizadas pelo imol de campo grande (MS). RBOL. 2017;4(1):46-52.

13.Carvalho CM, Nazar RJ, Moreira AMC, Bouchardet $\mathrm{FCH}$. Identificação humana pelo exame da arcada dentária. Relato de caso. Arq bras odontol. 2008;4(21):67-9.

14. Rawson RD, Ommen RK, Kinard G, Johnson J, Yfantis A. Statistical evidence for the individuality of the humam dentition. J Forensic Sci. 1984;29(1):245-53.

15.Bush AM, Bush PJ, Sheets HD. Statistical Evidence for the Similarity of the Human Dentition. Journal of forensic sciences, 2011;56(1):118-23.

16. Nambiar P, Carson G, Taylor JA, Brown KA. Identification from a bitemark in a wad of chewing gum. J Forensic Odonto-Stomatol. 2001;19(1):5-8.

17. Oliveira DCA, Simões PS, Marques JAM, Galvão LCC, Oliveira RN, Musse JO. Avaliação de marcas de mordidas em alimentos produzidas por próteses dentárias. Arq Odontol. 2010;46(1):38-42.

18. Marques JAM, Galvão LCC, Silva M. Marcas de mordidas. Feira de Santana: Universidade Estadual Feira de Santana; 2007.

19. Nascimento M, Sarmento VA, Beal VE, Galvão LCC, Marques JAM. Identificação de indivíduos por meio das marcas de mordida em alimentos utilizando a engenharia reversa e a prototipagem rápida: caso simulado. Arq Odontol. 
2012;48(3):134-41.

20. Figueira Júnior E, Moura LCL. A importância dos arcos dentários na identificação humana. Rev bras Odontol. 2014;71(1):22-7.

\section{CONFLITO DE INTERESSES}

Os autores declaram não haver conflitos de interesse.

AUTOR PARA CORRESPONDENCIA

\section{Cléa Adas Saliba Garbin}

clea.saliba-garbin@unesp.br

Submetido em 20/08/2018

Aceito em 04/10/2018 